\title{
Provider perspectives on barriers to reproductive health services for HIV-infected clients in Central Malawi
}

\author{
Margaret R Caplan ${ }^{1 *}$, Khumbo Phiri ${ }^{2}$, Julie Parent ${ }^{2}$, Ann Phoya ${ }^{3}$, Alan Schooley ${ }^{2,4}$ and Risa M Hoffman ${ }^{4}$ \\ ${ }^{1}$ Division of HIV Medicine, Los Angeles Biomedical Institute at Harbor-UCLA Medical Center, Torrance, California, USA \\ ${ }^{2}$ Partners in Hope, Lilongwe, Malawi \\ ${ }^{3}$ UNC Maternal and Safe Motherhood Program, Lilongwe, Malawi \\ ${ }^{4}$ Department of Medicine, Division of Infectious Diseases, David Geffen School of Medicine at UCLA, Los Angeles, California, USA
}

\begin{abstract}
Despite widespread availability of Depo-Provera in HIV clinics in Malawi, coverage of family planning (FP) remains low. We sought to understand provider perspectives about the challenges of providing reproductive health services to HIV-infected clients in antiretroviral therapy (ART) clinics in Central Malawi by conducting surveys and semi-structured in-depth interviews with 31 ART providers across 16 clinical sites. Additionally, site surveys were performed to assess contraceptive resources. Major barriers to the provision of FP in ART clinics were inadequate staff in the facility, shortage of trained providers, limited time to counsel on FP, and lack of private space for the provision of FP services. These barriers limit the direct delivery of FP in ART clinics. Strategies to integrate FP with HIV/ ART services and task shifting FP service provision to non-ART providers should be explored in Malawi as a means to improve coverage of services to HIV-infected clients.
\end{abstract}

\section{Introduction}

HIV/AIDS is the leading cause of death in women of childbearing age worldwide [1], and HIV-infected women carry an estimated 2 to 10 times higher risk of dying during pregnancy and the postpartum period compared to HIV-uninfected women [2-4]. Family planning (FP) can reduce morbidity and mortality in HIV-infected women and their families by preventing unplanned pregnancies and/or planning for healthy pregnancies with child-spacing and use of antiretroviral therapy (ART) [5-6]. Addressing FP and other reproductive health needs is essential to health promotion and disease prevention in HIV-infected women and men; however, despite the recognition of its importance, there are large gaps in the provision of FP services in Sub-Saharan Africa [7]. In a survey of $200 \mathrm{HIV}$-infected women presenting to an HIV/ART clinic in Lilongwe, Malawi, over two-thirds of the women reported that their last pregnancy was unintended and less than $10 \%$ were using hormonal contraception [8]. Understanding reasons for gaps in FP is important for effective implementation of services to improve HIV care.

Healthcare providers serve a critical role in the implementation of FP services for HIV-infected clients by determining access to and quality of services [9], and by influencing childbearing decisionmaking through positive and/or negative interactions with clients [10-11]. Our group initially performed a pilot study with 25 providers utilizing focus group discussions. The data suggested that providers have complex perspectives surrounding the issue of reproductive health services, possibly stemming from limited knowledge about risks of transmission of HIV to infants during pregnancy and lack of information about family planning and safer conception [12]. Building on our previous work, the current study performed individual in-depth interviews with HIV providers in Malawi who provide FP and ART services. We sought to better understand barriers and facilitators to the delivery of FP services among providers working in ART clinics in Central Malawi.

\section{Methods}

This mixed-method study was carried out in Central Malawi in September 2015 at 16 facilities supported by Extending Quality Improvement (EQUIP)-Malawi, a program funded by the President's Emergency Plan for AIDS Relief-United States Agency for International Development (PEPFAR-USAID) and implemented by Partners in Hope (PIH) Medical Center, a nongovernmental organization based in Lilongwe, Malawi. The study consisted of three components: (1) a brief socio-demographic survey of ART providers, (2) individual in-depth interviews with ART providers, and (3) a site survey to characterize FP services available at each facility including availability of contraception and frequency of stock outs of different types of contraceptives.

ART providers (physicians, clinical officers, medical assistants, and nurses) were eligible if they were 18 years of age or older and were actively providing care to HIV-infected clients at an EQUIP-supported facility. Convenience sampling was used to select 16 health facilities across four districts in Central Malawi. Informed consent was obtained from all individual participants included in the study. After obtaining oral informed consent, a university-trained Malawian researcher (K.P) with experience in conducting qualitative interviews administered the survey and in-depth interview in English, which is the official language of Malawi and known to healthcare providers through their training [13]. Survey questions focused on socio-demographic data

Correspondence to: Margaret R. Caplan, 1124 W. Carson Street, Bldg CDCRC Room 204, Torrance, CA 90502, USA, Tel: 1-424-571-7767; Fax: 1-310-7822964; E-mail: margaret.caplan@labiomed.org

Key words: reproductive health, family planning, HIV, contraception, Malawi

Received: January 20, 2018; Accepted: February 08, 2018; Published: February 12,2018 
and included questions about HIV clinical experience and training in reproductive health. In-depth interviews focused on several domains, including providers' knowledge about reproductive health, experiences and practices around service delivery of FP, barriers to the provision of $\mathrm{FP}$, and attitudes towards reproductive health service delivery as part of HIV care. Questions from the interview guide are provided in Table S1.

Survey data were completed on paper, tabulated, and summarized using Microsoft Excel (2013). In-depth interviews were audio recorded and transcribed. Qualitative data analysis was performed using Atlas. ti (version 1.0.31(117), Berlin, Germany). Two researchers reviewed the transcripts and used open coding to identify preliminary themes. Themes developed for the interview guide shaped the dominant themes, and open-ended questions enabled new themes and subthemes to emerge upon coding using the grounded approach [14]. These themes were then organized into over-arching, higher order concepts. Proportions of themes were calculated based on the number of occurrences in interviews. The study was approved by the Malawi National Health Sciences Research Committee and the University of California Los Angeles (UCLA) Institutional Review Board.

\section{Results}

Table 1 delineates the participating clinical sites and types of providers based on geography. The 16 clinical sites ranged in size from small rural health centers to large district hospitals. There was a median of 543 HIV-positive clients per site (range 92-5,700 clients). The median number of healthcare workers per site was 7 (range 2-122 providers), and the median number of HIV providers per site was 5 (range 2 to 47 providers).

All clinics had regular stocks of at least one type of hormonal FP, including combination oral contraception, Depo-Provera, and implants. Additionally, nearly half $(\mathrm{N}=7)$ stocked intrauterine devices (IUDs). Forty-four percent of sites $(\mathrm{N}=7)$ reported FP stock outs of at least one form of hormonal contraception in the last 6 months, and $43 \%(\mathrm{~N}=3)$ of these sites reported FP stock outs as a recurrent problem. Most of the stock outs were of Depo-Provera and male and female condoms. At the time of the survey, all sites reported having DepoProvera in stock; however, $30 \%(\mathrm{~N}=5)$ reported male condoms as out of stock and $25 \%(\mathrm{~N}=4)$ reported female condoms as out of stock. Fortyfour percent $(\mathrm{N}=7)$ of sites provided $\mathrm{FP}$ training to providers in the last 2 years, and all sites $(100 \%, \mathrm{~N}=16)$ had $\mathrm{FP}$ educational materials in the form of charts and posters in waiting areas and exam rooms. Although all ART programs provide FP as part of HIV care, none of the sites had a fully integrated or co-located FP program with dedicated staff and FP supplies readily available in the ART provider space.

Thirty-one providers were approached for participation in the study and all provided consent and completed study procedures. Table 2 summarizes participant characteristics and responses to survey questions about FP. Fifty-eight percent $(\mathrm{N}=18)$ of providers interviewed were female, and $65 \%(\mathrm{~N}=20)$ were nurses or nurse-

Table 1. Summary of clinical sites and healthcare providers.

\begin{tabular}{|l|c|c|c|}
\hline & Urban & Rural & Total \\
\hline Number of clinical sites visited & $\mathbf{4}$ & $\mathbf{1 2}$ & $\mathbf{1 6}$ \\
\hline Number of providers interviewed & $\mathbf{8}$ & $\mathbf{2 3}$ & $\mathbf{3 1}$ \\
\hline \multicolumn{1}{|c|}{ Clinical officer } & 2 & 1 & 3 \\
\hline Medical assistant & 0 & 8 & 8 \\
\hline Nurse/mid-wife technician & 6 & 14 & 20 \\
\hline
\end{tabular}

Table 2. Characteristics of healthcare providers interviewed $(\mathrm{N}=31)$.

\begin{tabular}{|c|c|c|}
\hline Characteristic & $\mathbf{n}$ & $(\%)$ \\
\hline Female gender & 18 & $(58 \%)$ \\
\hline \multicolumn{3}{|l|}{ Type of healthcare worker } \\
\hline Clinical Officer & 3 & $(10 \%)$ \\
\hline Nurse/Nurse-midwife Technician & 20 & $(65 \%)$ \\
\hline Medical Assistant & 8 & $(25 \%)$ \\
\hline \multicolumn{3}{|l|}{ Religion } \\
\hline Catholic & 5 & $(16 \%)$ \\
\hline Protestant & 21 & $(68 \%)$ \\
\hline Other Christian & 4 & $(13 \%)$ \\
\hline Muslim & 1 & $(3 \%)$ \\
\hline \multicolumn{3}{|l|}{ Days per week spent on HIV care: } \\
\hline$\leq 1$ & 1 & $(3 \%)$ \\
\hline$>1$ to 2 & 5 & $(16 \%)$ \\
\hline$>2$ to 3 & 0 & $(0 \%)$ \\
\hline$>3$ to 4 & 1 & $(3 \%)$ \\
\hline$>4$ to 5 & 24 & $(77 \%)$ \\
\hline \multicolumn{3}{|c|}{$\begin{array}{l}\text { Frequency of asking HIV clients about desires or } \\
\text { concerns about having children: }\end{array}$} \\
\hline All of the time & 6 & $(19 \%)$ \\
\hline Most of the time & 13 & $(42 \%)$ \\
\hline Some of the time & 12 & $(39 \%)$ \\
\hline Never & 0 & $(0 \%)$ \\
\hline \multicolumn{3}{|c|}{$\begin{array}{l}\text { Frequency of asking HIV clients about family planning } \\
\text { other than condoms: }\end{array}$} \\
\hline All of the time & 20 & $(65 \%)$ \\
\hline Most of the time & 6 & $(19 \%)$ \\
\hline Some of the time & 4 & $(13 \%)$ \\
\hline Never & 1 & $(3 \%)$ \\
\hline Prior training in family planning, y & 26 & $(84 \%)$ \\
\hline Family planning materials" at site, $y$ & 29 & $(94 \%)$ \\
\hline \multicolumn{3}{|l|}{ Depo-Provera stock outs in last 90 days: } \\
\hline Never & 24 & $(77 \%)$ \\
\hline 1 week or less & 3 & $(10 \%)$ \\
\hline$>1$ week & 0 & $(0 \%)$ \\
\hline Don't know & 4 & $(13 \%)$ \\
\hline
\end{tabular}

"Examples include posters, videos, booklets, and handouts.

midwife technicians (NMT). Eighty-four percent of providers $(\mathrm{N}=26)$ reported having training in FP in the past, and all providers $(100 \%$, $\mathrm{N}=31$ ) reported asking their HIV-positive clients about fertility desires "at some point," although only $19 \%(\mathrm{~N}=6)$ asked at every routine visit.

\section{Provider views of reproductive health and family planning service delivery}

Almost all providers $(94 \%, \mathrm{~N}=29)$ described reproductive health and family planning as important issues for both men and women. In response to asking "Please define what the term family planning means to you", $42 \%(\mathrm{~N}=13)$ reported that it is a choice made by an individual about having children. One provider stated:

"This can be defined as, it's a choice...that is done by women, men, girls, boys, within reproductive age, on how to, and when maybe to choose to have children, how many children, how far they should be spacing their children." -35 year-old female, nurse, rural site

Forty-five percent $(\mathrm{N}=14)$ of providers defined $\mathrm{FP}$ as a choice made by a couple or family. One medical assistant stated:

"Family planning? Is a process whereby a man and a woman and their family agrees...to have a number of children, and in doing that, they choose a type of contraceptive that will help them to have the 
number of children they want and at specific intervals."-34 year-old male, medical assistant, rural site

In response to the question "What method of contraception is best for HIV-infected individuals?" 52\% ( $\mathrm{N}=16)$ answered Depo-Provera. Half of these $(\mathrm{N}=8)$ also mentioned dual protection with Depo-Provera and condoms as the optimal method. Thirty-five percent of providers $(\mathrm{N}=11)$ reported "long-term" or "permanent" methods as the best strategy, referring to IUDs and sterilization.

\section{Provider-perceived barriers to provision of family planning}

Health system barriers: Among providers interviewed, provision of FP services was generally not limited by availability of medications but by structural issues such as lack of integration with ART services and lack of clinical capacity. When asked to describe "the biggest barrier to providing FP options to HIV-infected clients," most providers $(91 \%, \mathrm{~N}=28)$ reported systems issues (Figure 1) and none of the providers reported stock out of contraceptives as a major barrier. The most common systems barrier cited by $45 \%(\mathrm{~N}=14)$ of providers was inadequacy of human resources, either not having enough staff or not having enough time for counseling clients. A medical assistant from a rural clinic working as the only non-nurse provider at the facility reported:

"Even in our setting, we have a good number [of] providers but sometimes we see that there's only one person working, so that is a great problem. [The] very same person has to ask [about HIV and family planning], which means I have to make sure that I have my Depo here and I have to inject them, at the very same time, I have 40 patients waiting outside... The contraceptive method must be near me, in this office. They shouldn't be in this closet."-34 year-old male, medial assistant, rural site

A clinical officer at another rural site reported organization of the space as a problem, with supplies in a different location from the provider's clinical space:

"[When] you provide these services far away from where the ART is... In that case, it becomes difficult for you to leave your [office] and go to the other room."-45 year-old male, clinical officer, rural site

Ten percent $(\mathrm{N}=3)$ noted lack of private space to administer FP services, including counseling and delivery of contraceptives:

"At this facility, I can say there's a barrier because we don't have a specific room where we can be providing these methods because our clinic is small." -29 year-old female, nurse officer, urban site
Providers $(\mathrm{N}=3)$ raised concern that when ART and FP services are offered on different days, clients do not utilize both services because of the cost of transport and amount of time required for each clinical visit. One provider reported improvement in this issue at his facility due to better coordination of services:

"We were doing [FP] on Tuesdays...they are supposed to come the same week maybe twice to the same clinic. Today [is] Thursday, we come to acquire only ART... Next Tuesday they will come for family planning. But now it's quite simple to give such a service [FP] to an ART client [on the same day]...previously it was a challenge."-29 year-old male, medical assistant, rural site

Providers suggested better integration of ART and FP could be a way to overcome common barriers. A medical assistant stated:

"So it's like you have a good space where you can do ART and provide the family planning methods all together. I think that could... ease the provision of these services. And also, maybe, to have more staff being trained in ART and in family planning, such that the one who is doing the services should be capable of doing both of these services at once.."-31 year-old male, medical assistant, rural site

Lack of training and knowledge around reproductive health: Lack of training in FP was cited as the "biggest" barrier by $26 \%$ of providers $(\mathrm{N}=8)$. One medical assistant acknowledged her own limitations:

"I've not had formal training in family planning so it becomes a barrier because I only know two types of methods to provide to the clients, like the condoms and the Depo-Provera, but I cannot provide other methods."-32 year-old female, rural site

Many providers have limited knowledge or misconceptions about reproductive health in HIV-infected clients, and this manifests as inaccurate counseling. In describing prevention of mother-to-child transmission (PMTCT), 16\% $(\mathrm{N}=5)$ of providers reported the risk of HIV transmission to a seronegative partner or child as being " $50 / 50$ " with or without intervention:

"I think those women who have got HIV-positive... they are not supposed to have children because the chances are, I can say the chances are 50/50 to some women [of passing HIV to the infant]."-34 year-old female, NMT, rural site

"It's good for me to counsel the others or the fathers who ask, who have come for ART, counsel them for the family planning methods

- Percentage of Interviewees ( $\mathrm{N}=31$ )

$10 \%(N=3)$

Lack of space/privacy

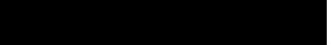

Inadequate time for counseling

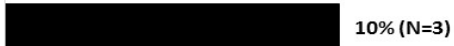

Inadequate number of staff

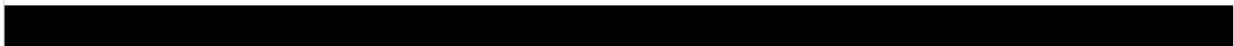

$35 \%(N=11)$

Lack of provider training

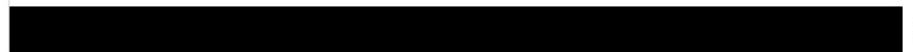

$26 \%(N=8)$

Not providing all methods

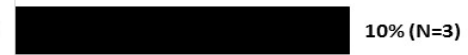

Figure 1. Factor identified as the biggest barrier to family planning in HIV-infected clients. 
because of the same reason-the risk of HIV infection to the baby during pregnancy... So all that said, it's 50/50."-40 year-old female, nurse, rural site

In terms of safer conception, only one provider was aware of ART and timing ovulation as a strategy for prevention of HIV to serodiscordant partners attempting conception. This nurse reported:

"If a [discordant] couple comes to me with such a request, I'm going to advise them. If they want to have children, we should also see about the viral load, especially if the viral load is low, the viral load is undetectable, I think that's a good time to have children. After they succeed and after they, as soon as they, conceive, they should continue using condoms."-38 year-old female, nurse, urban site

Provider personal beliefs and family planning: Providers believe childbearing is a human right but often carry judgment about whether individuals with HIV should have children. The idea of childbearing as a human right emerged in $16 \%(\mathrm{~N}=5)$ of interviews.

"I also told him that it's their right to have children. No one cannot tell them that...we cannot advise them to not have children 'cause it's their life. But we just tell them about the consequences if they are not adhering to drugs." -32 year-old female, medical assistant, rural site

One medical assistant from a rural clinic recognized a shift in risk with the development of PMTCT and safer conception strategies:

"Yeah, of course, they have the right to bear children... when I was joining the ART provision services, we were strongly advising these people not to bear any more children with HIV status. But, I think this time around things have changed. Women are bearing children with HIV status. The good thing is that the children are born free of HIV. I think it's their right to bear children. But not frequent. They have to be at least spacing, not less than three years in between."-42 year-old male, medical assistant, rural clinic

Twenty-nine percent of providers $(\mathrm{N}=9)$ reported trying to give information about health risks while remaining impartial in the client's decision.

"You know, in normal cases, we don't decide for them. They have to decide. We just give them the information."-45 year-old male, clinical officer, urban site

"...so long as we give them information, much information, yeah, it's their choice. We don't deceive them, but it's a choice, I can't say it's good or bad but it's a choice of the individual after give information."-35 year-old female, nurse, rural site

"As a provider, I'm very comfortable in providing this information to the clients because ... whether positive or negative, they have to be treated equally."-42 year-old, male, medical assistant, rural site

Seventy-one percent $(\mathrm{N}=22)$ of providers raised opinions that individuals with HIV should limit childbearing. Interviews revealed a tension between provider's views that reproduction is a human right and provider's concern about health risks for HIV-infected clients having children.

"Well it depends on the woman, if she chooses to have children, we are there to guide her because to say if somebody's HIV-positive, it's better not to have children but because they have that right to say, 'I should have children' who are we to say no...As usual, I tell them that it's better not to have a child because you never know."-45 year-old male clinical officer, rural site
Providers recognized cultural norms and community pressures around childbearing and appeared to be more accepting of risks of conception for newly married couples or couples with no children.

"...my understanding that for a woman who is HIV positive and she doesn't have children... it's proper for her to have at least one child because culturally, in our culture, there's this myth that if you don't have a child, it means maybe the marriage, it will be, again, a problem. But, again, on the other hand if the woman is HIV infected, if she has at least a child or two..., I don't think it's proper for that particular woman, again, to be getting pregnant ...."-42 year-old male, NMT, rural site

\section{Discussion}

In our study, providers viewed system-level issues such as human resources, time for counseling, and privacy as major barriers to the FP delivery in the context of HIV care. Providers identified integration of ART and FP services as a means of addressing many of these barriers within the confines of the current system. Published conceptual models highlight the overarching importance of system-level factors in the provision of reproductive healthcare to HIV-infected clients by shaping patient decision-making through information and support given, shared provider attitudes, and availability of risk reduction services [15].

In conceptualizing research and evaluation priorities, Kendall et al. identified integrated health service delivery of reproductive health and HIV services as a key priority for reducing preventable HIV-related maternal mortality in Sub-Saharan Africa [16]. Studies demonstrating the benefits of integrated HIV and reproductive health services have largely focused on incorporating HIV testing, counseling, and treatment into existing maternal and child health $(\mathrm{MCH})$ services rather than incorporating FP and other reproductive health services into longitudinal HIV care and treatment [17-18]. Experiences from the $\mathrm{MCH}$ setting have shown that system adaptations are needed for effective integration. On an operational level, providers of integrated HIV and postnatal services in Kenya reported increased service uptake by clients; however, they also reported significant occupational stress related to increased workload and limited time for counseling patient [19]. Researchers at the Lighthouse Trust in Lilongwe, Malawi, recently published their experiences in a pilot study of integrated sexual and reproductive health services and FP into routine HIV care at one clinic site without additional staffing [18]. They note that this integration is feasible with the utilization of task shifting to re-distribute workload and recommend FP training of HIV clinic staff, including refresher training, ongoing staff support, and open discussions to dispel myths about FP. Of note, as part of the process of integration they performed a construction project creating private exam and counseling rooms at an estimated cost of USD 70,000.

Limited knowledge and misconceptions about FP and safer conception were a barrier to provision of accurate, unbiased counseling for providers in our study. Knowledge of the importance of ART in PMTCT was common; however, very few providers were aware of ART as a means of preventing partner transmission and fewer knew about timed ovulation and/or self-insemination. As expected, providers who expressed confidence in knowledge of recent advancements in safer conception tended to be more objective in counseling HIV-infected clients about how to safely have children. The association between increased knowledge and the ability to provide objective reproductive health counseling was also observed in our previous study using focus 
groups with HIV providers in Malawi [12].

Providers in our study shared the belief that childbearing is a human right, while also expressing judgment about whether individuals with HIV should have children, particularly for couples who already have one or more children. Most providers recognized the importance of childbearing to their clients and its value as a cultural norm, and appeared to struggle between supporting the client's fertility desires and counseling on strategies that carry any level of risk for adverse outcomes for the client, partner, and/or child. We observed similar tension in our previous study using focus groups with providers in Malawi, in which there was recognition of reproductive rights, but a reluctance to counsel due to concerns about risks of transmission to the partner and the child, and concerns that HIV-infected individuals may not be able to care for children due to chronic HIV [12].

These findings are also similar to a qualitative study in Uganda published in 2016, also focusing on provider perspective of safer conception services for HIV-infected clients. In this study, providers expressed discomfort raising the option of condomless sex to achieve pregnancy even in the setting of ART and discouraged pregnancy because of perceived risks of HIV transmission and poor health outcomes of mother and child [20]. The authors highlight that providers lacked training in safer conception and struggled to offer reproductive strategies that might be associated with any risk of HIV transmission. These findings are particularly striking in the era of expanded ART, data on treatment as effective prevention, and studies showing near normal lifespan for individuals who start therapy early. Taken together, these studies suggest a need for more education and training on FP and safer conception that include data-driven approaches that reinforce low risk strategies, engage providers in a dialogue about reproductive health rights, and dispel myths and misconceptions about HIV-infected clients having children.

\section{Limitations}

This was a qualitative study designed to explore provider perceptions of FP and reproductive health barriers in our program, with the majority of facilities being rural and located within Central Malawi. Thus, the overall generalizability of the results may be limited. All facilities in which we conducted interviews were providing FP, although none were a formally integrated or co-located service within ART; therefore, this study may not have captured the opinions of providers working at facilities where FP is fully integrated. Although we utilized a researcher who was not known to providers as a colleague, participants may have been reluctant to share views because the evaluation project was carried out by an organization supporting care and treatment at the facilities involved in the study. Additionally, interviews were performed by a female, and male providers may have been uncomfortable presenting their views on gender, family planning, and reproductive health to a female interviewer.

\section{Conclusion}

HIV providers view system-level issues such as human resources, time for counseling, and privacy as major barriers to the delivery of FP in the context of HIV care. Integration of ART and FP may address these barriers but would require additional adjustments such as task shifting and identification of private spaces within the ART clinic where service delivery can occur. Models of care that offer comprehensive reproductive health services for all individuals across a facility and/ or catchment area should also be evaluated and could strengthen FP services broadly, rather than solely for HIV-infected clients. Additionally, in order to effectively deliver comprehensive reproductive health services, providers require training that emphasizes the safety and efficacy of modern contraception and safer conception strategies and includes dialogue about reproductive health rights and unbiased counseling. The opportunity to strengthen FP and reproductive health care for HIV-infected clients will allow for optimizing outcomes for these individuals and for building healthy families and communities.

\section{Acknowledgements}

We gratefully acknowledge the providers at EQUIP-supported sites across central Malawi who participated in this project. We are thankful to the Lilongwe-based EQUIP-Malawi staff for providing administration and oversight for this project. This research was made possible with support from funding provided by the President's Emergency Plan for AIDS Relief (PEPFAR) through USAID-Malawi under the terms of Grant No. 674-A-00-10-00035-00; and M.C. received support from the National Institute of Mental Health at the National Institutes of Health under Grant No. 5T32MH80634-09.

\section{Compliance with ethical standards}

Conflict of Interest: The authors declare that they have no conflict of interest.

Ethical Approval: All procedures performed in studies involving human participants were in accordance with the ethical standards of the institutional and/or national research committee and with the 1964 Helsinki declaration and its later amendments or comparable ethical standards.

Informed Consent: Informed consent was obtained from all individual participants included in the study.

\section{References}

1. WHO. Women's Health. Fact sheet $\mathrm{N}^{\circ} 334$. September 2013.

2. Le Coeur S, Halembokaka G, Khlat M, Brouard N, Purhuence F, et al. (2005) Impact of AIDS on adult mortality: a morgue-based study in Pointe-Noire, Republic of Congo. AIDS 19: 1683-1687. [Crossref]

3. Calvert C, Ronsmans C (2013) The contribution of HIV to pregnancy-related mortality: a systematic review and meta-analysis. AIDS 27: 1631-1639. [Crossref]

4. Lathrop E, Jamieson DJ2, Danel I2 (2014) HIV and maternal mortality. Int J Gynaecol Obstet 127: 213-215. [Crossref]

5. Zijenah L, Mbizvo MT, Kasule J, Nathoo K, Munjoma M, et al. (1998) Mortality in the first 2 years among infants born to human immunodeficiency virus-infected women in Harare, Zimbabwe. J Infect Dis 178: 109-113. [Crossref]

6. Marinda E, Humphrey JH, Iliff PJ, Mutasa K, Nathoo KJ, et al. (2007) Child mortality according to maternal and infant HIV status in Zimbabwe. Pediatr Infect Dis $J 26$ : 519-526. [Crossref]

7. Jacobstein R, Curtis C, Spieler J, Radloff S (2013) Meeting the need for modern contraception: effective solutions to a pressing global challenge. Int J Gynaecol Obstet 121 Suppl 1: S9-15. [Crossref]

8. Lisa B. Haddad, Caryl Feldacker, Denise J. Jamieson, Hannock Tweya, Carrie Cwiak, et al. (2015) Pregnancy prevention and condom use practices among HIV-infected women on antiretroviral therapy seeking family planning in Lilongwe, Malawi. PLoS One 10: $\mathrm{e}$ 0121039. [Crossref]

9. Anand A, Shiraishi RW, Bunnell RE, Jacobs K, Solehdin N, et al. (2009) Knowledge of HIV status, sexual risk behaviors and contraceptive need among people living with HIV in Kenya and Malawi. AIDS 23: 1565-1573. [Crossref]

10. Shelton J (2001) The provider perspective: human after all. Int Fam Plan Persepct 27: 152-155.

11. Myer L, Morroni C, El-Sadr WM (2005) Reproductive decisions in HIV-infected individuals. Lancet 366: 698-700. [Crossref]

12. Kawale P, Mindry D, Phoya A, Jansen P, Hoffman R (2015) Provider attitudes about 
childbearing and knowledge of safer conception at two HIV clinics in Malawi. Reprod Health 12: 17. [Crossref]

13. Chien E, Phiri K, Schooley A, Chivwala M, et al. (2016) Successes and Challenges of HIV Mentoring in Malawi: The Mentee Perspective. PLoS One 11: e0158258. [Crossref]

14. Corbin J, Strauss A (1990) Grounded theory method: Procedures, canons, and evaluative criteria. Qualitative Sociology 13: 3-21.

15. Crankshaw TL, Matthews LT, Giddy J, Kaida A, Ware NC, et al. (2012) A conceptual framwework for understanding HIV risk behvior in the context of supporting fertility goals among HIV-serodiscordant couples. Reprod Health Matters 20(39 suppl): 50-60. [Crossref]

16. Kendall T, Danel I, Cooper D, Dilmitis S, Kaida A, et al. (2014) Eliminating Preventable HIV-Related Maternal Mortality in Sub-Saharan Africa: What Do We Need to Know? $J$ Acquir Immune Defic Syndr 67(Suppl 4): S250-S258. [Crossref]
17. Lindegren ML, Kennedy CE, Bain-Brickley D, Azman H, Creanga AA, et al. (2012) Integration of HIV/AIDS services with maternal, neonatal and child health, nutrition, and family planning services. Cochrane Database Syst Rev 12: CD010119. [Crossref]

18. Phiri S, Feldacker C, Chaweza T, Mlundira L, Tweya H, et al. (2016) Integrating reproductive health services into HIV care: strategies for successful implementation in a low-resource HIV clinic in Lilogwe, Malawi. J Fam Plann Reprod Health Care 42: 17-23. [Crossref]

19. Mutemwa R, Mayhew S, Colombini M, Busza J, Kivunaga J, et al. (2013) Experiences of health care providers with integrated HIV and reprodcutive health services in Kenya: a qualitative study. BMC Health Serv Res 13: 18. [Crossref]

20. Matthews LT, Bajunirwe F, Kastner J, Sanyu N, Akatukwasa C, et al. (2016) "I Always Worry About What Might Happen Ahead": Implementing Safer Conception Services in the Current Envrionement of Reproductive Counseling for HIV-Affected Men adn Women in Uganda. Biomed Res Int. [Crossref]

Copyright: $\mathbb{C} 2018$ Caplan MR. This is an open-access article distributed under the terms of the Creative Commons Attribution License, which permits unrestricted use, distribution, and reproduction in any medium, provided the original author and source are credited. 